\title{
PARTICLE-BASED FLOW VORTICITY ANALYSIS BY USE OF SECOND-GENERATION WAVELETS
}

\author{
ODDNY H. BRUN ${ }^{1}$, JOSEPH T. KIDER JR. ${ }^{1} \&$ R. PAUL WIEGAND ${ }^{2}$ \\ ${ }^{1}$ School of Modeling, Simulation, and Training University of Central Florida, USA \\ ${ }^{2}$ Department of Computer Science and Quantitative Methods, Winthrop University, USA
}

\begin{abstract}
Modeling, simulating, and analyzing turbulent flow is a topic of high interest from both a verification and accuracy aspect. This work presents computational methods and experimental measures of turbulent fluid flow modeled with particle-based smoothed particle hydrodynamics (SPH), as well as the use of second-generation wavelets to analyze the nature of vorticity. Modeling and analyzing vorticity by use of first-generation wavelets for regular grid methods are well presented in literature. We are unaware of any work on this topic for particle-based methods. The difference between regular grid-based and particle-based approaches are due to irregularities introduced by the latter. We found that secondgeneration wavelets proved to be robust, fast, and reliable. Second-generation wavelets are designed to handle irregular grids and do not rely on a dyadic number of observations, which make them suitable candidates for SPH analysis as opposed to first generation wavelets. The resulting significant discrete wavelet transform (DWT) coefficients are found to be representative of the flow sections that may benefit from additional attention in the simulation model. The robustness of the method allows for fast initial screening of the flow to highlight sections that are of interest for more detailed analysis. Here, robustness refers to the two parameters significance level and grid resolution. Our results are demonstrated using a 2D sloshing tank case.
\end{abstract}

Keywords: smoothed particle hydrodynamics (SPH), vorticity, second-generation wavelets, thresholding.

\section{INTRODUCTION}

Real-time simulations of flow models depend on fast and efficient methods for analyzing the vast amount of data typically generated during a simulation run. In this work, we show how a method based on second-generation wavelets was used to analyze the data from a smoothed particle hydrodynamics (SPH) simulation of less smooth flow. While the irregular particle spacing in SPH represents welcoming flexibility it has also proven to be a challenge to the method from the very beginning and still continues to be (Monaghan [1]). The degree of irregularity increases for turbulent flow and hereby challenges the smoothing process that is a fundamental step towards achieving accuracy (Rafiee et al. [2]). The work presented here demonstrates how a second-generation wavelet approach is successfully used to:

- efficiently identify the sections of the flow of relevance for improvement and

- handle SPH generated data of irregular structure.

As pointed out by numerous researchers, among others Farge et al. [3], flow vorticity is an important parameter in less smooth flow modeling. We apply second-generation wavelet analysis to the vorticity values of our flow simulation data. As far as we have been able to verify, no such approach has been presented before. Previous work presented on flow analysis using wavelets is based on first-generation wavelets data on regular grid structures. Firstgeneration wavelets require a dyadic number of observations and a regular grid structure. For particle-based methods, including SPH, these conditions are not met. Hence, the motivation for using second-generation wavelets, which do not require either of those two conditions. With the second-generation wavelet method, we were able to quickly and efficiently identify 
the sections of the flow where the focus of vorticity analysis is expected to be beneficial. In section two of this paper, the methods are presented. The results of our analysis are presented in section three. Data from the SPH simulation of a two-dimensional sloshing tank case was used. Concluding remarks are presented in section four.

\section{THE FUNDAMENTALS IN TERMS OF METHODS AND CONDITIONS}

In this section, we have included methods and conditions of specific relevance to the work presented in this paper. For the fundamentals of fluid dynamics, including smoothed particle hydrodynamics (SPH) and wavelet theory, the reader is encouraged to seek the relevant sources on those topics. Example of such sources are Monaghan [1], Lucy [4], Mallat [5], Monaghan [6]. The relevance of vorticity analysis in turbulent fluid modeling is thoroughly discussed by Farge [7], Farge et al. [8] as well as several later publications by the same group of researchers. The motivation for use of wavelets is based on properties related to orthogonal and biorthogonal wavelets that provide a decomposition of the flow field into independent components (Farge et al. [3], Urban [9], Urban [10]).

The difference between first- and second-generation wavelets of relevance to vorticity analysis lies in the fact that a first-generation wavelet can be chosen so that its base functions are spanned by a Riesz basis, meaning it is orthogonal or close to orthogonal, and hence provide an independent component split. Second-generation wavelets do not fulfill the conditions of a Riesz basis, which leads to two concerns: stability and lack of independence in the decomposition. The stability concern is remediated by a check and balance to ensure low condition numbers leading to stable basis functions as presented by Vanraes et al. [11], [12]. The lack of independence in our case was expected to be ignorable as the purpose of our analysis was of the nature of feature extraction. Roussel et al. [13] quantified the effect of biorthogonal wavelets to a loss of three percent in enstrophy compared to orthogonal when analyzing a 3D homogeneous turbulent flow, something one should take into consideration if the results were to be used in an operational step of the simulations with influence on mass, momentum, or energy conservation. Under such conditions, a further quantification of the lack of independence would be required. The nature of second-generation wavelets lets the basis functions adapt to the irregularity of the data (Jansen and Oonincx [14], Sweldens [15]), which is part of why orthogonality is not achievable.

The nature of vorticity in turbulent flow is assumed to be comprised of a coherent and an incoherent component. The coherent component is characterized by stronger and further reaching correlation as opposed to the incoherent component that takes the nature of Gaussian white noise. The separation of the two components is achieved by applying a threshold to the coefficients produced by a discrete wavelet transform. This mechanism is the same as what has been known for decades for signal or data denoising. The specifics of selecting the thresholding level is among others described by Donoho and Johnstone [16] and Abramovich et al. [17] as examples of hard and soft thresholding, respectively. We used a hard threshold in accordance with earlier work on turbulence analysis. This threshold, $t_{c u t}$, was calculated as:

$$
t_{c u t}=\sqrt{\frac{2}{3} \sigma_{c D}^{2} \ln (n)}
$$

where $\sigma_{c D}^{2}$ is the DWT coefficients' estimated variance and $n$ is the number of coefficients. Multiple previous works have used alternative ways to calculate the estimate, one being the variance of all coefficients, another being the variance of the smaller coefficents, referred to respectively as high and low thresholding in the presentation of our results below. The low threshold is calculated with an iterative process and halted when the difference in variance between two consecutive iterations becomes rather small. The reasoning behind this approach 
is based on the fact that white noise variance is constant. We found that in most cases four iterations were enough to achieve convergence.

We would in general recommend that a thresholding mechanism should be selected depending on the purpose and sensitivity of the analysis. This is based on results from earlier work (Brun [18]) where soft thresholding resulted in more accurate results than hard when used for discontinuity identification purpose.

The open-source software DualSPHysics (Domínguez et al. [19]) was used for our simulations and the discrete wavelet transforms were performed by the code for secondgeneration wavelets analysis provided in MATLAB MathWorks [20] with a symmlet wavelet. The technical details of second-generation wavelets are beyond the scope of this paper, however, open access to MATLAB source code for reproduction purposes will be made available at a later time.

\section{RESULTS}

In this section, we present the major findings and implications of vorticity analysis by secondgeneration wavelets. The major findings were:

- The nature of vorticity in particle-based flow simulations were identifiable by the use of second-generation wavelets.

- The coherent vorticity structures were separated from the incoherent ones by the significant discrete wavelet transform (DWT) coefficients.

- The method demonstrated robustness in terms of resolution level needed to locate the coherent structures in the flow.

- A relatively high threshold for significance was sufficient to identify the location of the stronger coherence. A lower threshold based on the white noise variance may be used to capture more details.

- A coarse resolution structure relative to average particle distance was able to identify the location of the stronger coherence. A finer resolution closer to average particle distance provided a more accurate location of the coherent structures. A resolution equal to initial particle spacing was insufficient for wavelet analysis.

- Combining high threshold and coarse resolution structure allowed for a fast approach to identify the sections of the flow where the stronger coherence were located.

- The irregular particle structure that particle based method represents was remediated by the second-generation wavelets.

- The method is memory efficient and fast due to the nature and technique used for the so-called lifting scheme combined with second-generation wavelets.

The results were demonstrated on a $2 \mathrm{D}$ sloshing tank case simulated by the smoothed particle hydrodynamics (SPH) method with the following case setup:

A rectangular body of water, initially located at the entire lower part of a tank, gets exposed to gravitational force and an additional external force represented by a sine wave causing the tank to rock back and forth over the y-axis (Fig. 1). The setup was with an initial average particle distance of $d p=0.001 \mathrm{~m}$ which resulted in 82,708 fluid particles. Results were sampled at $40 \mathrm{kHz}$. The simulation ran for 8.35 seconds.

\subsection{Coherent vs. non-coherent vorticity separated by second-generation wavelet transform}

The vorticity analysis was performed on a time frame basis by applying the discrete wavelet transform (DWT) to the particles' vorticity values. The distinction between non-significant 


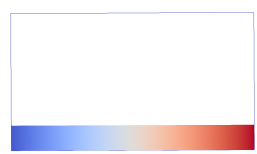

(a) Time zero second.

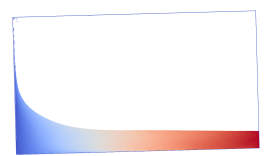

(b) Time frame 1,019.

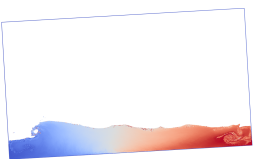

(c) Time frame 1,600 .

Figure 1: Sloshing motion case, shown at initial stage, $t=0$, at $t=1,019, \sim 2.56 \mathrm{sec}$, and at $t=1,600, \sim 4.02 \mathrm{sec}$. Colors are based on initial particle position, that is: particles positioned left are blue, to the right are red.

and significant DWT coefficients was obtained by applying a threshold, which meant setting the DWT coefficients with absolute value less than the threshold level to zero while keeping unchanged the DWT coefficients of absolute value higher than the threshold level. The resulting significant DWT coefficients appeared in the sections of the flow where coherent vorticity structures were present. An example from time frame 1, 600, 4.02 seconds into the simulation mon is nracanted in Fir ? The amount and lomations of coherent vorticity seem is observed when comparing

\section{Significant DWT}

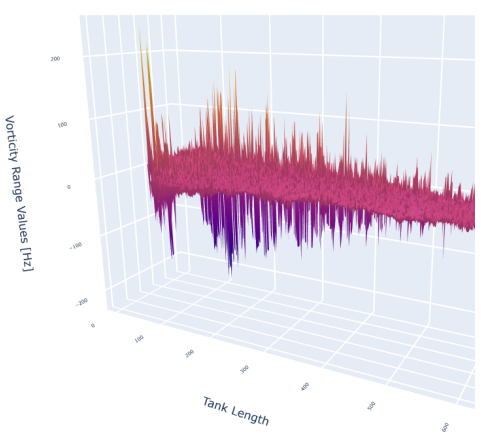

(a) Vorticity value:

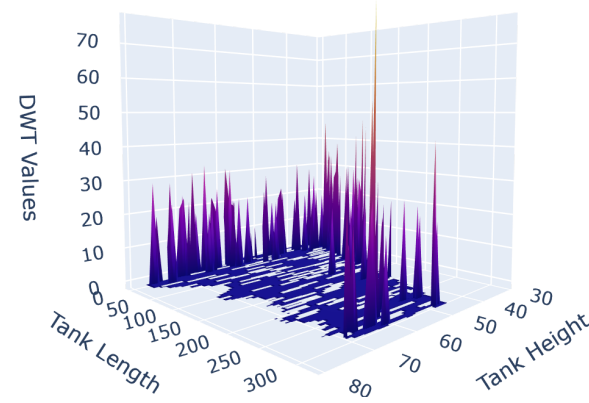

(b) Significant DWT coefficients.

Figure 2: Vorticity values and significant DWT coefficients indicating coherent vorticity at $t=1,600, \sim 4.02 \mathrm{sec}$.

\subsection{Robustness}

The vorticity analysis based on second-generation wavelet analysis demonstrated robustness both in terms of threshold levels as well as resolution used for the flow field. The work presented on regular grid-based vorticity analysis have used the approach of both a higher 


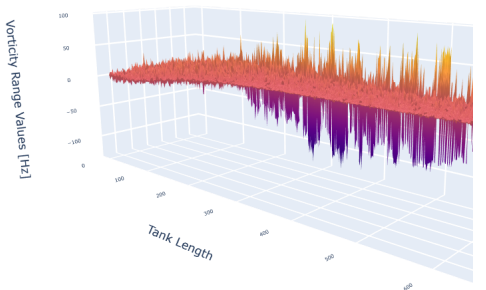

(a) Vorticity value:

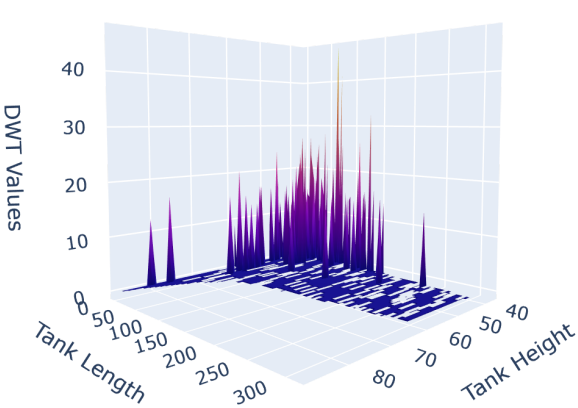

(b) Significant DWT coefficients.

Figure 3: Vorticity values and significant DWT coefficients indicating coherent vorticity at $t=1,019, \sim 2.56 \mathrm{sec}$.

threshold based on the total flow field variance as well as a threshold based on the white noise variance estimate which leads to a lower threshold. We found the higher threshold resulted in just above three percent significant DWT coefficients while the lower threshold resulted in just above eleven percent when comparing results from one and the same time frame.Figs 4 and 5 both visually and quantitatively demonstrate the difference.In general, the selection of threshold should depend on the purpose of the analysis. With a regular grid and first-generation wavelet analysis, it has been reported that the higher threshold represented a somewhat lower level of enstrophy compared to if the lover threshold was used Farge et al. [8], Okamoto et al. [21]. We did observe a similar effect as we performed an inverse discrete wavelet transform (IDWT) of the significant DWT coefficients and compared the results by taking the $l_{2}$ norm. The higher cut resulted in about two percent lower $l_{2}$ norm of the reproduced vorticity values than the lower cut. For the purpose of the work here, our

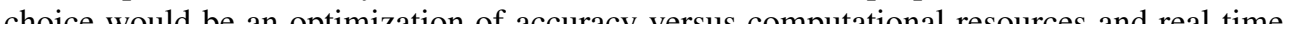

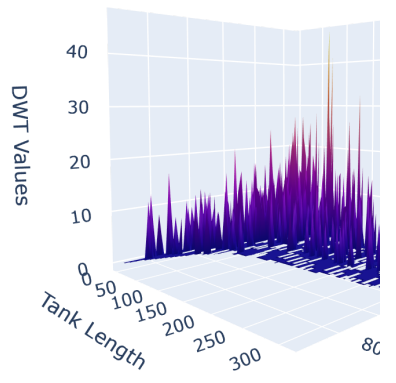

(a) Low thresho

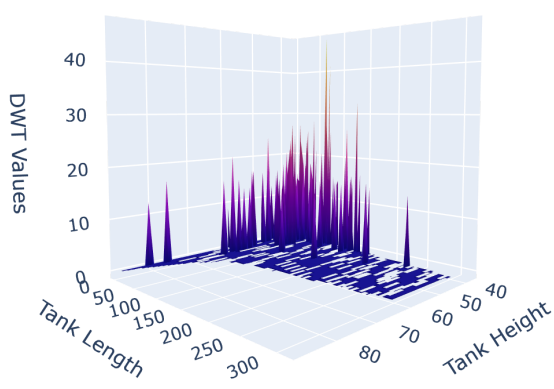

(b) High threshold.

Figure 4: Threshold robustness. Significant DWT coefficients indicating coherent vorticity values at $t=1,019, \sim 2.56 \mathrm{sec}$ of 3.5 and 11.0 percent, respectively. 


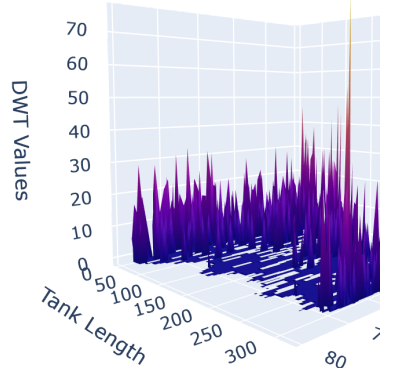

(a) Low thresho

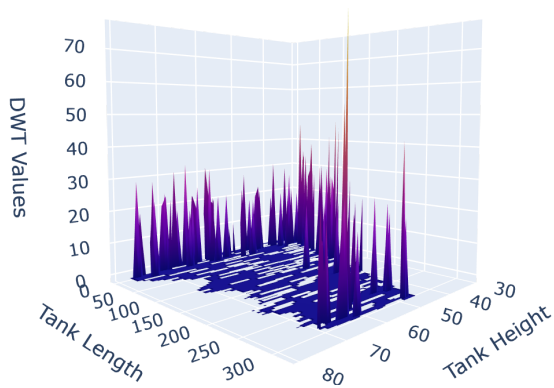

(b) High threshold.

Figure 5: Threshold robustness. Significant DWT coefficients indicating coherent vorticity values at $t=1,600, \sim 4.02 \mathrm{sec}$ of 3.1 and 11.0 percent, respectively.

The setup leading to the results presented so far was based on a resolution structure of 0.0013 by $0.0013 \mathrm{~m}$, where the $0.0013 \mathrm{~m}$ is the radius of the circle defining the smoothing function's compactly supported area for our 2D SPH based case. A finer resolution equivalent to the initial particle spacing of $0.001 \mathrm{~m}$ resulted in too sparse particle representation proving insufficient for the wavelet analysis. However, more coarse structures equivalent to two and three times the $0.0013 \mathrm{~m}$ did identify significant DWT coefficients located in the sections that were already identified by use of the higher resolution as shown in Fig. 6(a) and 6(b), respectively. Further, the reduction of the resolution level by the factors two and three slightly increased the percentage of significant DWT coefficients to 4.1 and 4.8 , respectively. This increase may partly have been caused by the fact that for lower resolution the vorticity range value for each cell was used as opposed to the actual vorticity value used at the higher resolution. The robustness of this method both in terms of resolution and threshold level demonstrated the potential of using such an approach to identify the sections of the flow that represent strong coherence. By that, the sections that have the higher potential of improved smoothing may be located in a fast and efficient manner.

\subsection{Particle irregularity}

In SPH, matter is represented in terms of particles where each particle carries its parameters, which typical are mass, position, acceleration, velocity, pressure, density, and vorticity. Additional particle characteristics may be added. The particles are initially in a regular equally-spaced arrangement. As soon as any force is applied to the matter, the particles are no longer equally-spaced. This loss of regularity is observed by a varying number of particles located within a section of given size. For a section of the flow with size equal to the compactly supported domain of the smoothing function used in a particular SPH simulation, the number of particles may vary from zero to several times the number of the initial arrangement. Fig. 7 shows multiple white areas, which means no particles are present in those parts of the flow field. The flow field itself is also of irregular shape with rugged edges. The second-generation wavelet transform was able to handle both those factors, something that would prohibit analysis by a first-generation wavelet. 


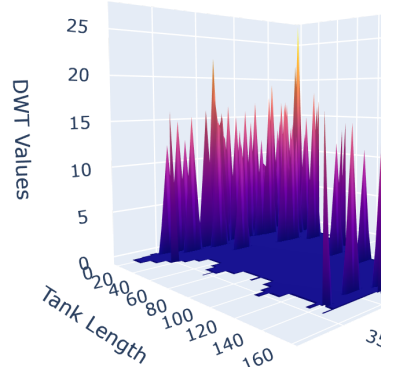

(a) Resolution 0.0026 by

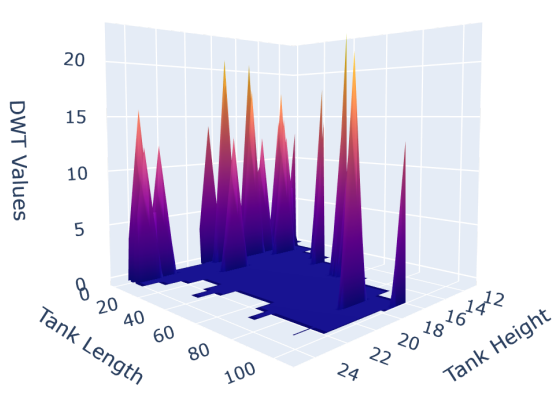

(b) Resolution 0.0039 by $0.0039 \mathrm{~m}$.

ndicating coherent vorticity ively, using a high threshold

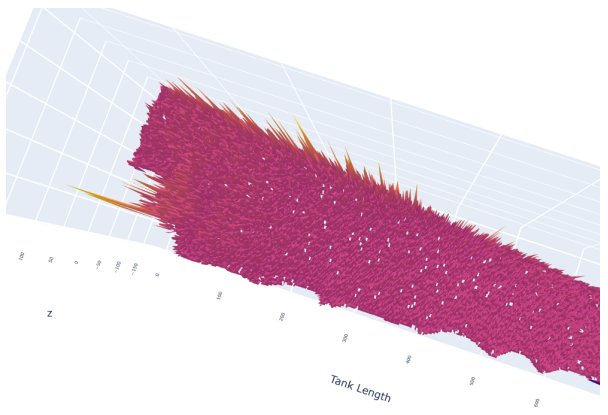

(a) Vorticity values.

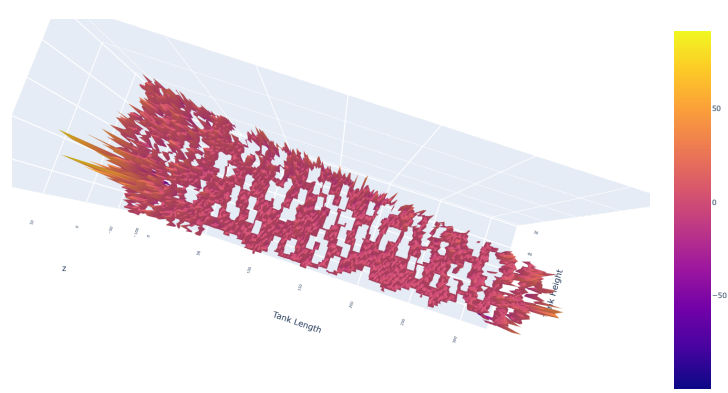

(b) DWT coefficients uncut.

Figure 7: Top view of vorticity values and DWT coefficients at $t=2,896, \sim 7.24 \mathrm{sec}$ where white sections within the flow field are sections with no particles present.

\subsection{Fast and memory efficient}

The amount of data generated by simulating the 2D sloshing tank case according to the setup described in the beginning of this section, a run was in the order of 82,708 fluid particles times 3,340 time frames times the number of parameters tracked per particle. With such a vast amount of data, efficient feature extraction tools are necessary. Our particular case was motivated by the need to separate the less smooth sequences into those where improvement (such improvements are outside the scope of this paper) was expected to have significant impact in terms of accuracy and those that could be neglected. Each of the 82,708 fluid particles represents such a smoothing sequence. Of those, about 25 to 28 percent represent less smooth sequences. The vorticity analysis by use of second-generation wavelets did enable us to reduce the number of particles relevant for further attention to about three 
58 Computational Methods and Experimental Measurements XX

percent, as well as identifying their location in the flow field. Fig. 8(a) and 8(b) demonstrate the feature extraction achieved. Other factors that contributed to speed and memory efficiency were:

nc

on of

thi

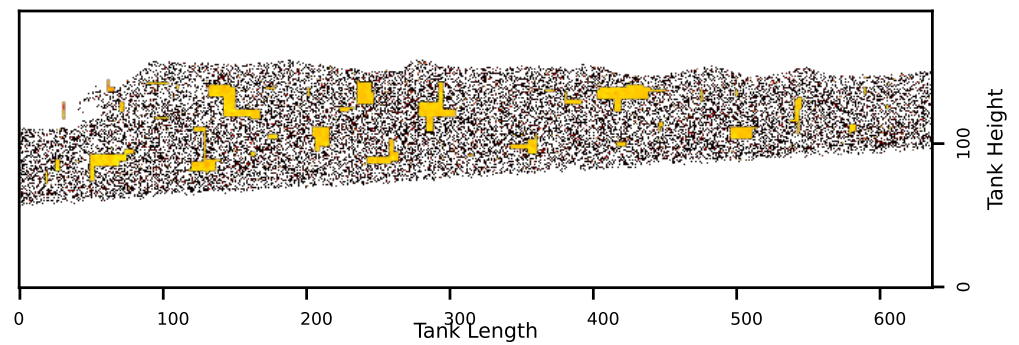

(b) At $t=2,896,3.0$ percent sign. coeffs.

Figure 8: Location of coherent vorticities (orange/yellow) compared to discontinuous smoothing sequences (black) for sloshing motion. Tank is divided into cells of 0.0013 by $0.0013 \mathrm{~m}$. 


\section{CONCLUSION}

The result of the work presented here shows the second-generation wavelets' ability to handle irregular data structures and efficiently extract key features of interest from vast amount of non-dyadic data. This was demonstrated by carrying out vorticity analysis of a two-dimensional turbulent flow simulated by smoothed particle hydrodynamics. The differentiation between the significant and non-significant discrete wavelet transform coefficients separated the coherent vorticity components and the incoherent ones. Earlier work states that the coherent components are characterized by stronger and further reaching correlation, so being able to locate those components is of high importance when optimizing were to focus on model improvements under turbulent flow conditions. We found the method robust and reliable as far as we were able to compare to vorticity analysis results by firstgeneration wavelets on regular grids. The method may have limitations in terms of accuracy as it does not produce orthogonality between the coherent and incoherent components. Such a limitation may be relevant in case the results were to be used as part of a model where the ability to conserve mass, momentum, or energy was influenced. This was not a concern in our work as the feature extraction effect was the goal of our analysis and we achieved a reduction in sections of relevance by a factor of about nine.

\section{REFERENCES}

[1] Monaghan, J. J., Particle methods for hydrodynamics. Computer Physics Report, 1985.

[2] Rafiee, A., Cummins, S., Rudman, M. \& Thiagarajan, K., Comparative study on the accuracy and stability of sph schemes in simulating energetic free-surface flows. European Journal of Mechanics, B/Fluids, 36, pp. 1-16, 2012.

[3] Farge, M., Schneider, K. \& Kevlahan, N., Non-gaussian and coherent vortex simulation for two-dimensional turbulence using an adaptive orthogonal wavelet basis. Physics of Fluids, 1999.

[4] Lucy, L.B., A numerical approach to the testing of the fission theory. The Astronomical Journal, 82, pp. 1013-1024, 1977.

[5] Mallat, S., A Wavelet Tour of Signal Processing The Sparse Way, Academic Press, 2009.

[6] Monaghan, J.J., Sph without a tensile instability. Journal of Computational Physics, 2000.

[7] Farge, M., Wavelet transforms and their applications to turbulence. Annual Review of Fluid Mechanics, 1992.

[8] Farge, M., Schneider, K. \& Kevlahan, N.K.R., Coherent structure eduction in waveletforced two-dimensional turbulent flow. IUTAM Symposium on Dynamics of Slender Vortices, eds E. Krause \& K. Gersten, 1998.

[9] Urban, K., Wavelet basis in h(div) and h(curl). Mathematics of Computing, 2000.

[10] Urban, K., Wavelets in Numerical Simulation, Springer, 2002.

[11] Vanraes, E., Jansen, M., \& Bultheel, A., Stabilised wavelet transforms for nonequispaced data smoothing. preprint Signal Processing stabwt, 2000.

[12] Vanraes, E., Jansen, M., \& Bultheel, A., Stabilised wavelet transforms for nonequispaced data smoothing. Signal Processing, 2002.

[13] Roussel, O., Schneider, K., \& Farge, M., Coherent vortex extraction in 3d homogeneous turbulence: Comparison between orthogonal and biorthogonal wavelet decomposition. Journal of Turbulence, 2005.

[14] Jansen, M. \& Oonincx, P., Second Generation Wavelets and Applications, Springer, 2010.

[15] Sweldens, W., The lifting scheme: A construction of second generation wavelets. SIAM Journal on Mathematical Analysis, 1998. 
[16] Donoho, D.L. \& Johnstone, I.M., Adapting to unknown smoothness via wavelet shrinkage. Journal of the American Statistical Association, 90, pp. 1200-1224, 1995.

[17] Abramovich, F., Benjamini, J., Donoho D.L. \& Johnstone, I. M. Adapting to unknown sparcity by controlling the false discovery rate. The Annals of Statistics, 34, pp. 584653, 2006.

[18] Brun, O.H. Improved interpolation in sph in cases of less smooth flow. Master's thesis, Institute for Simulation and Training, University of Central Florida, 2016.

[19] Domínguez, J.M., Crespo, A.J.C. \& Rogers B.D., Users guide for dualsphysics code, 2016. https://dual.sphysics.org/.

[20] MathWorks, Lifting method for constructing wavelets. 2021. https://www.mathworks.com/help/wavelet/ug/lifting-method-for-constructingwavelets.html.

[21] Okamoto, N., Yoshimatsu, K., Schneider, K., Farge, M. \& Kaneda, Y., Coherent vortices in high resolution direct numerical simulation of homogeneous isotropic turbulence: A wavelet viewpoint. Physics of Fluids, 2007.

[22] Hastings, E.J., Mesit, J. \& Guha, R.K., Optimization of large-scale, real-time simulations by spatial hashing. Summer Simulation Conference, 2005.

[23] Mallat, S.G., Multifrequency channel decompositions of images and wavelet models. IEEE Transactions on Acoustics, Speech, and Signal Processing, 1989.

[24] Sweldens, W., The lifting scheme: A custom-design construction of biorthogonal wavelets. Applied and Computational Harmonic Analysis, 1996. 